\title{
Aneurysm Coil Embolization Using a I.5-Fr Distal Outer Diameter Microcatheter
}

\author{
David A. Stidd, MD, MS, Demetrius K. Lopes, MD, Michael Chen, MD
}

The current assortment of microcatheters widely used for aneurysm coil embolization may not be well suited for several anatomic variants, including excessive vascular tortuosity and small aneurysms less than $3 \mathrm{~mm}$. Longer microcatheters designed with a smaller caliber that can accommodate and deliver coils may be of use in these situations. This case series and literature review illustrates the advantages and limitations of the Marathon microcatheter (Covidien, Irvine, CA, USA) when used for coil embolization of cerebral aneurysms. Despite some technical compromises including the distal marker, length, and the risk of buckling, the Marathon microcatheter was able to adequately deliver coils to achieve satisfactory occlusion of cerebral aneurysms. We found unique advantages with regards to length and smaller distal outer diameter (OD). These results may serve as a guide for the further development of a microcatheter used for coil embolization that has the features of a smaller distal OD and longer length.

Key Words : Cerebral aneurysms; Coil embolization; Endovascular procedures; Microcatheter

The distal outer diameter (OD) of the majority microcatheters currently being used for aneurysm coil embolization are all at least 1.6-Fr and most are not longer than $150 \mathrm{~cm}$ in length. There has been interest in the use of larger caliber microcatheters to deliver larger coils with the potential advantage of delivering coils that are softer and achieve greater volumetric filling [1]. However, there are anatomic situations whereby a smaller caliber and longer microcatheter may be of benefit. We present a case series evaluating the use of a

\footnotetext{
All authors: Department of Neurosurgery, Rush University Medical Center, Chicago, IL, USA

Received January 15, 2014; accepted after revision February 17, 2014.

Correspondence to: Michael Chen, MD, Rush University Medical Center, 1725 W. Harrison Street, Suite 855 Chicago, Illinois 60612, USA

Tel. 1.312.563.2817 Fax. 1.312.942.2206

E-mail: Michael_Chen@rush.edu

This is an Open Access article distributed under the terms of the Creative Commons Attribution Non-Commercial License (http://creativecommons.org/licenses/by-nc/3.0) which permits unrestricted non-commercial use, distribution, and reproduction in any medium, provided the original work is properly cited.
}

Marathon microcatheter (Covidien, Irvine, CA, USA) for aneurysm coil embolization and discuss the advantages, limitations and potential implications.

\section{CASE PRESENTATION}

\section{Case 1}

A bilobed anterior communicating artery (ACoA) aneurysm in an 80-year-old man with a transient ischemic attack five years prior to presentation was noted to have aneurysm growth up to $7 \mathrm{~mm}$ in maximal diameter on serial imaging. During an elective endovascular embolization procedure, severe tortuosity was noted at the femoral iliac junction during guide catheter access, as well as at the origin of the left common carotid artery, which shared an origin with the innominate off the aorta. The final guide catheter access consisted of a $90 \mathrm{~cm}$ length multipurpose tip shape Neuron Max 088 (Penumbra Inc, Alameda, CA, USA) guide catheter to the left distal common carotid artery over a $115 \mathrm{~cm}$ length Navien 058 (Covidien Neurovascular, Irvine, CA, USA) intermediate guide catheter to the left distal cervical internal carotid artery 
David A. Stidd, et al.

(ICA). Both guide catheters were "hubbed-out" or inserted entirely into the patient. Attempts to advance a $150 \mathrm{~cm}$ length SL-10 (Stryker Neurovascular, Fremont, CA) microcatheter over a wire only allowed access to the A1 segment of the left anterior cerebral artery, whereby the hub of the microcatheter was in contact with the hub of the intermediate catheter.

A $165 \mathrm{~cm}$ Marathon microcatheter with a distal inner diameter (ID) of 0.013 " instead was advanced over a Synchro 10 guidewire (Stryker Neurovascular,
Fremont, CA, USA) and access to the dome of the aneurysm was obtained (Fig. 1A). A $5 \mathrm{~mm} \times 13$ $\mathrm{cm}$ Barricade coil (Blockade Medical, Irvine, CA, USA) was then advanced easily and smoothly through the Marathon microcatheter. The pusher wire length was insufficient to completely deploy the coil, necessitating removal of the rotating hemostatic valve (RHV), and therefore the continuous flush to the microcatheter. This shorter length was sufficient to completely deploy the coil with enough pusher wire length to attach a
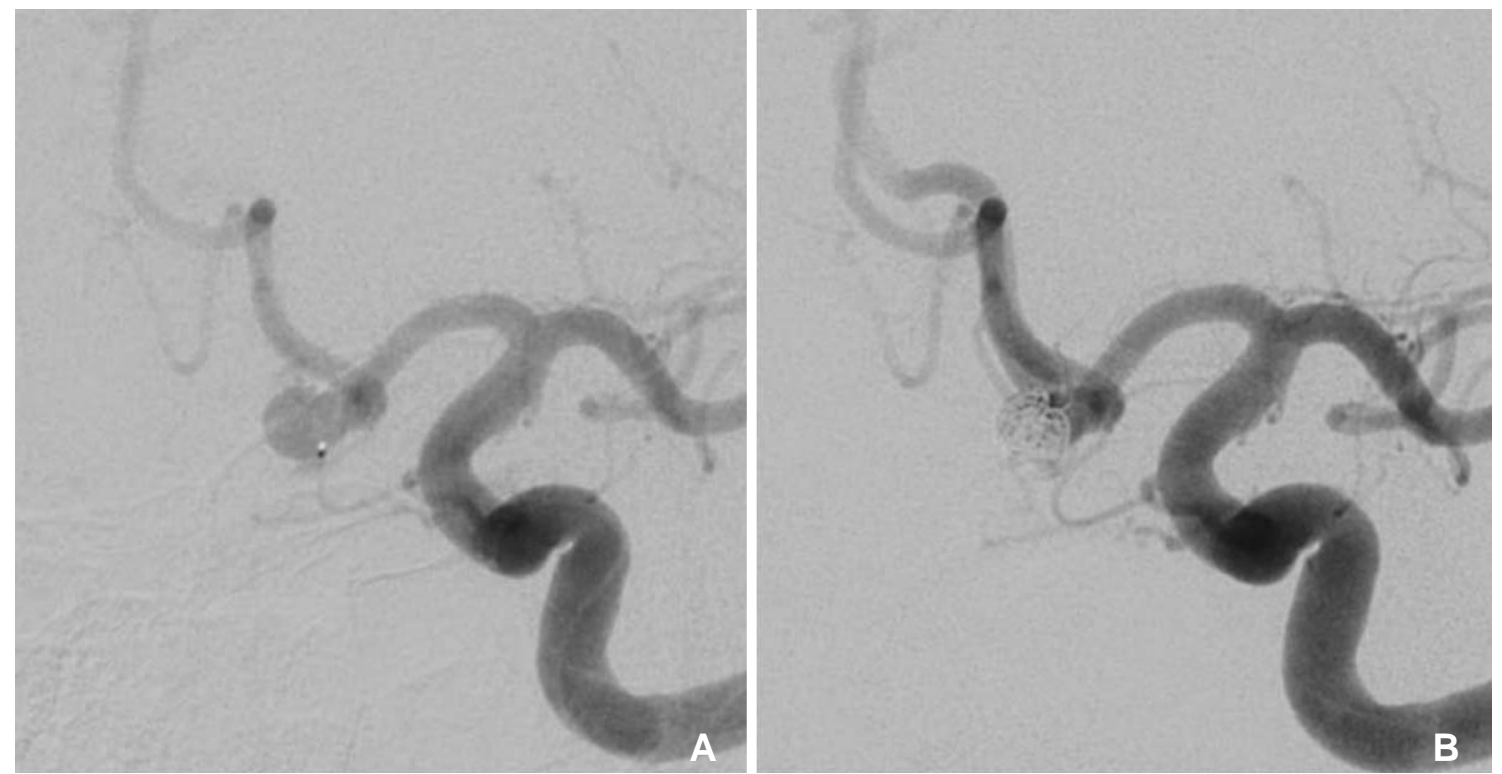

Fig. 1. Coil embolization of an ACoA aneurysm using a Marathon microcatheter. A. Selective catheterization of the $6 \mathrm{~mm}$ bilobed $\mathrm{ACoA}$ aneurysm from the left ICA with a Marathon microcatheter. B. Satisfactory aneurysm coil embolization using $32 \mathrm{~cm}$ of bare platinum coil with uniform distribution.
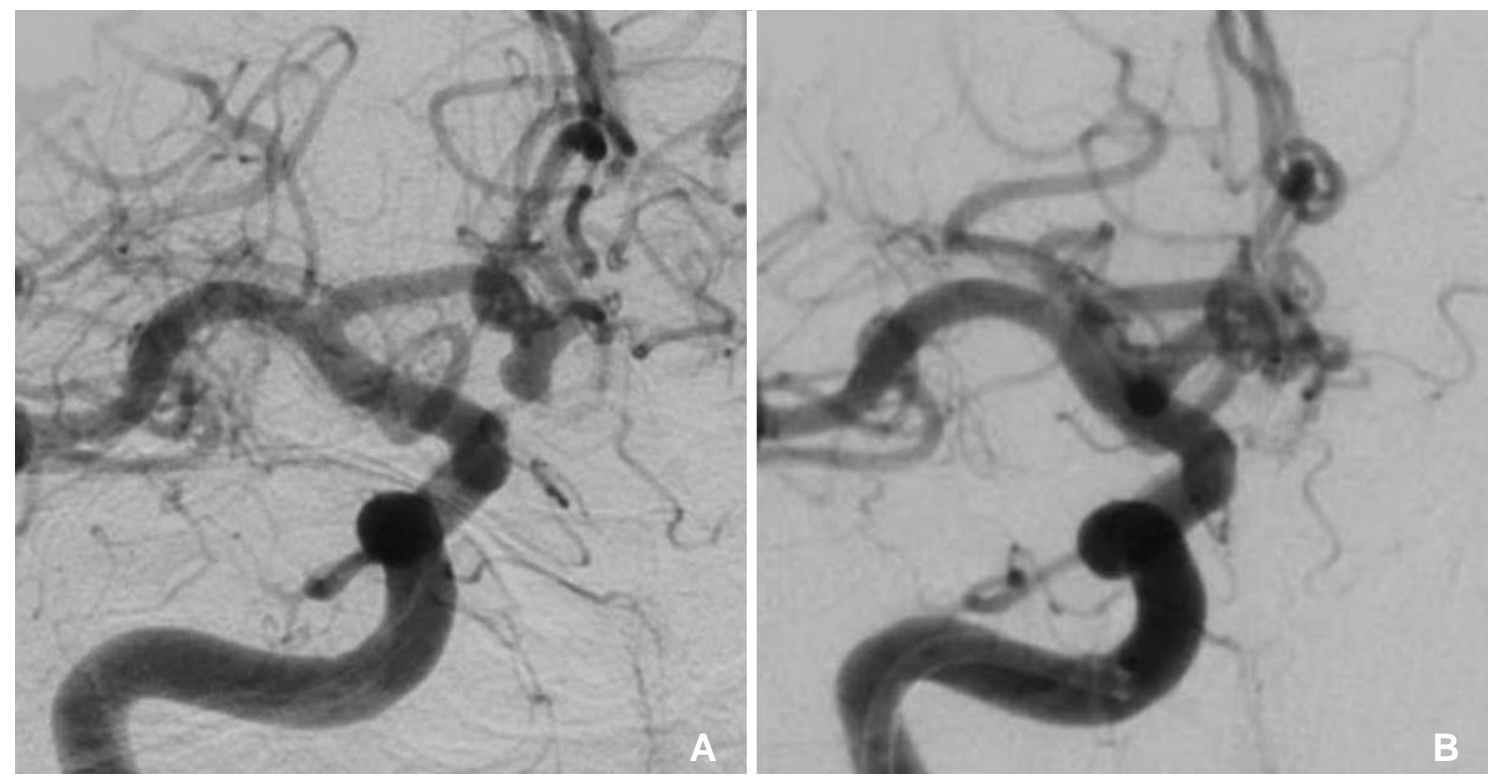

Fig. 2. Lateral arterial phase angiogram of a right ICA injection. A. $4 \mathrm{~mm}$ inferiorly directed ruptured ACoA aneurysm. B. Raymond I occlusion using $14 \mathrm{~cm}$ of bare platinum coil. 


\section{5-Fr OD Microcatheter Used for Coil Embolization}

circuit clip to electrolytically detach the coil. Detachment was also complicated by the lack of a proximal radio-opaque marker on the distal aspect of the Marathon microcatheter which by convention is located $3 \mathrm{~cm}$ from the most distal tip of other catheters used to deploy coils. This required additional time to slowly and carefully advance the pusher wire until

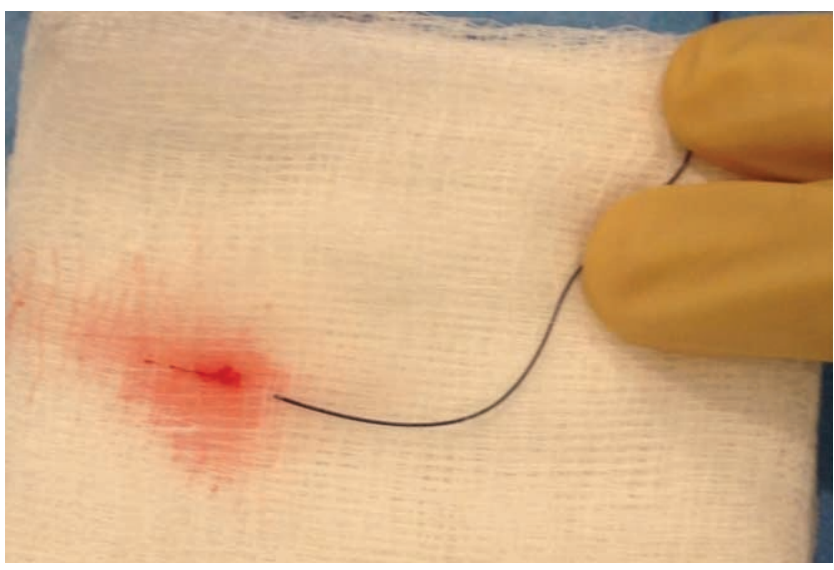

Fig. 3. Clot flushed from the first Marathon microcatheter removed during Case 2 after the coil was withdrawn from the microcatheter. detachment zone was out of the microcatheter and detachment actually occurred. Five coils in total were deployed, including a $5 \mathrm{~mm} \times 13 \mathrm{~cm}, 4 \mathrm{~mm} \times 7 \mathrm{~cm}$, $3 \mathrm{~mm} \times 6 \mathrm{~cm}, 2.5 \mathrm{~mm} \times 6 \mathrm{~cm}$ and a $2.5 \mathrm{~mm} \times 4 \mathrm{~cm}$. The final coil was able to achieve a satisfactory occlusion grade and the microcatheter was removed without issue (Fig. 1B). The patient tolerated the procedure well and was discharged home the next day with a normal neurologic examination.

\section{Case 2}

A 48-year-old man was admitted with a severe headache and subarachnoid hemorrhage in the suprasellar cistern, interhemispheric fissure, and bilateral sylvian fissures. He was found to have a $5 \mathrm{~mm}$ bilobed, inferiorly directed ACoA aneurysm (Fig. 2A). Using a 6-Fr Neuron guide catheter, access was obtained to the right cervical ICA. A Marathon microcatheter was advanced over a Synchro 10 guidewire to selectively catheterize the aneurysm. Upon withdrawal of the wire, there was some movement of the microcatheter tip that required adjustment of forward tension on the microcatheter. Coil
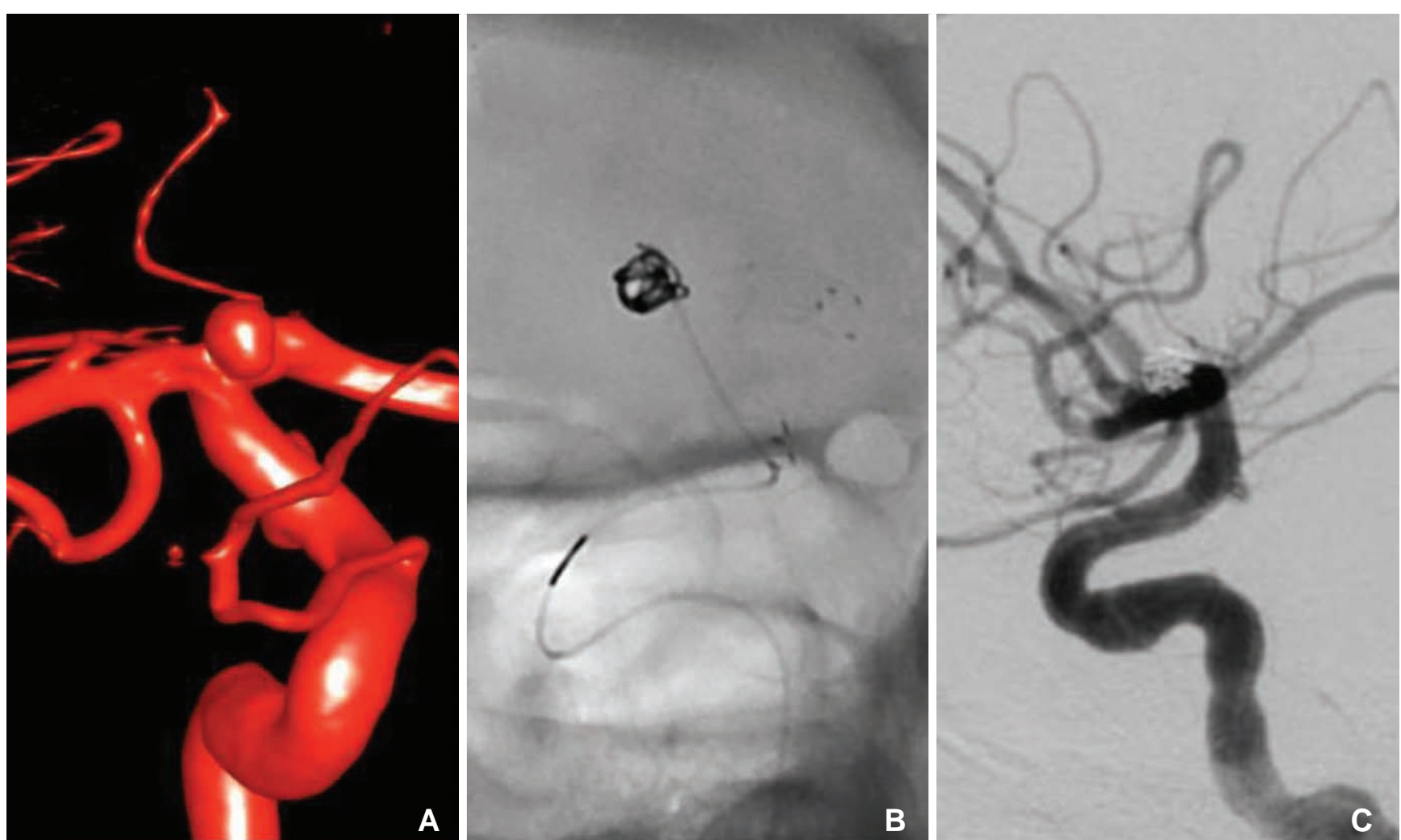

Fig. 4. Stent-assisted coil embolization of a right ICA terminus aneurysm. A. A surface reconstruction of 3D DSA data acquired from a right ICA injection. B. Fluoroscopic image of the coil embolization of the ICA aneurysm showing a Marathon microcatheter aneurysm catheterization through a Neuroform stent deployed from the ICA to the A1 segment. The 3-cm maker is visible on the pusher wire, but a corresponding 3-cm radioopaque marker is not present on the Marathon microcatheter. C. Raymond I occlusion of the ICA terminus aneurysm. 


\section{David A. Stidd, et al.}

embolization with a $3 \mathrm{~mm} \times 6 \mathrm{~cm}$ and $3 \mathrm{~mm} \times 4 \mathrm{~cm}$ Barricade coils proceeded with caution. As in the previous case, the RHV and hence continuous flush had to be removed to accommodate the complete deployment of the coils. The lack of a $3 \mathrm{~cm}$ distal marker on the Marathon microcatheter prolonged the detachment times since caution was necessary to avoid advancing the pusher wire out of the distal microcatheter tip. Upon placement of a third $2 \mathrm{~mm} \times$ $4 \mathrm{~cm}$ coil, there appeared to be increased resistance within the microcatheter causing buckling of the microcatheter near the carotid siphon. This was presumably because of clot collecting within the microcatheter. The microcatheter was removed, and after straightening out the catheter, the undetached coil was retrieved. The microcatheter was flushed on the back table, and clot emerged from the microcathter (Fig. 3). Another Marathon microcatheter was then advanced into the aneurysm and coil embolization was completed without complication (Fig. 2B). The patient tolerated the procedure well and was discharged home after an uneventful hospital stay with a normal neurologic examination.

\section{Case 3}

A 48-year-old female with a strong history of familial cerebral aneurysms was found to have a $4 \mathrm{~mm}$ saccular aneurysm with a broad neck at the right ICA terminus on a screening MRA of the brain. The patient was consented for an elective stent-assisted coil embolization of the aneurysm (Fig. 4) given the family history a ruptured cerebral aneurysms and patient preference. On

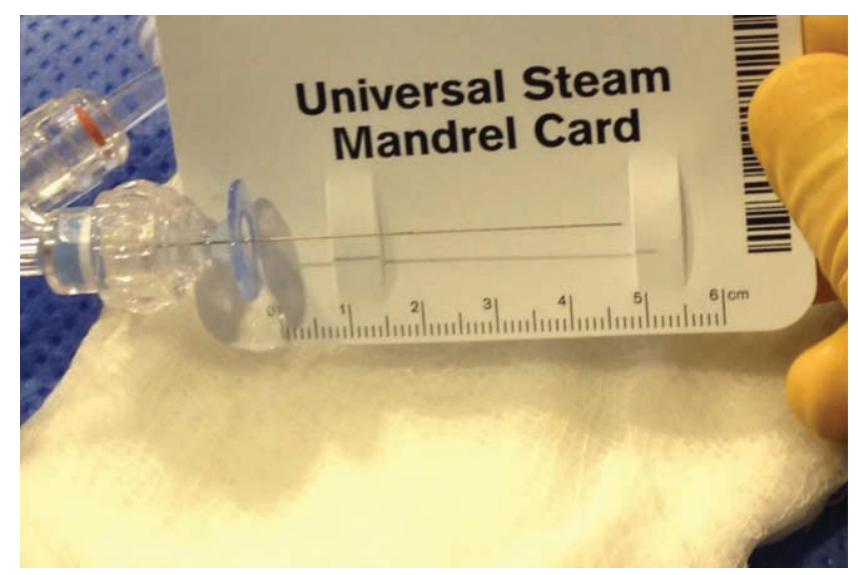

Fig. 5. Photograph showing only $5 \mathrm{~cm}$ of pusher wire extending from the RHV attached to the Marathon microcatheter from Case 3. The Target coil was fully deployed into the aneurysm, but there was not enough pusher wire left to attach the handheld electrolytic detachment device. The RHV had to be removed from the Marathon microcatheter first before the detachment device could be used to detach the coil into the aneurysm. the day of the procedure, the right common carotid artery was selected with a 6-Fr Neuron guide catheter through a right common femoral artery access. A 4.5 $\times 20 \mathrm{~mm}$ Neuroform EZ stent (Stryker, Kalamazoo, MI, USA) was deployed from the A1 segment into the ICA. A Marathon microcatheter was used to catheterize the ICA terminus aneurysm through the stent and a 3 $\mathrm{mm} \times 6 \mathrm{~cm}$ and $2 \mathrm{~mm} \times 4 \mathrm{~cm}$ Target Ultrasoft coils (Stryker, Kalamazoo, MI, USA) were deployed through the Marathon catheter without complication. During deployment of each coil, the RHV and thus continuous flush was temporally removed to accommodate the Styrker electrolytic detachment device (Fig. 5). After the coils were detached from the pusher wire, the electrolytic detachment device was removed and the RHV was replaced restoring the continuous flush before the pusher wire was withdrawn from the catheter to avoid clot formation within the microcatheter. The patient tolerated the procedure well and later discharged home neurologically intact.

\section{DISCUSSION}

Factors traditionally thought to impact in vivo microcatheter performance include lubricity, stiffness and durability [2]. Microcatheters may be broadly divided into reinforced and non-reinforced devices. The Marathon microcatheter was designed as a flow directed microcatheter to deliver DMSO and Onyx liquid embolic agent to typically distal arterial pedicles of a brain arteriovenous malformation. The design consists of a single distal tip radio-opaque marker, and the total usable length is $165 \mathrm{~cm}$. A nitinol braid in the distal segment of the microcatheter creates a floppy, soft end capable a safely catheterizing small delicate vessels and stainless steel coil reinforcement in the proximal shaft gives the microcatheter stiffness.

Deploying coils through a Marathon microcatheter has several advantages compared to other conventionally used microcatheters for aneurysm coil embolization. Most notably is the longer usable length, which may be needed at times for severe vascular tortuosity. In the first case, the hubs of the microcatheter, intermediate catheter, and guide catheter were all in contact, and the $150 \mathrm{~cm}$ length standard coiling microcatheter was too short to access the ACoA aneurysm. In such situations, a direct arteriotomy at the cervical carotid artery for intracranial vascular access has been reported [3]. The option of exchanging the entire system such that the guide catheter was removed and only the 115 $\mathrm{cm}$ intermediate catheter was in place was another 


\section{5-Fr OD Microcatheter Used for Coil Embolization}

possible option. Using another microcatheter with an extra $15 \mathrm{~cm}$ of microcatheter length proved to be a much more straightforward solution to the challenges associated with vascular tortuosity.

Increased "paint-brushing" action, or the back-andforth movement of the microcatheter tip as the coil was being inserted, was noted during the cases allowing for more uniform distribution of coils within the aneurysm. This facilitated more uniform coil placement and less chance of compartmentalization. In third case, the tip of the Marathon advanced rather easily through the interstices of the Neuroform stent, which may be another advantage.

Technical challenges we found in using the Marathon for coil embolization included the absence of a proximal $3 \mathrm{~cm}$ coil-detachment marker. Particularly in situations whereby the tip of the microcatheter is not well visualized, such as when it is within a nest of coils, there may be a risk of advancing the pusher wire outside of the microcatheter and potentially injuring the aneurysm wall. Horie et al have demonstrated that the Marathon microcatheter can be used to deploy detachable coils, but suggest that only Electro Detach Coils (Kaneka, Osaka, Japan) can be used because the Marathon only has one distal marker [4]. We show that the detachment process is safely performed with other coils, but may take more time as serial attempts to detach the coil are made as the coil is gradually and in a stepwise fashion advanced out of the microcatheter.

Another challenge with this technique is the mismatch of the Marathon microcatheter length and the length of the coil and pusher wire, precluding the use of a RHV and therefore the use of continuous flush in the microcatheter during detachment. In our cases, only about $3 \mathrm{~cm}$ of coil pusher wire were exposed even with the RHV removed. This short pusher wire exposure

Table 1. Comparison of Relevant Dimensions Among 10-size Microcatheters Used for Aneurysm Coil Embolization

\begin{tabular}{lccc}
\hline Microcatheter & $\begin{array}{c}\text { Distal ID } \\
\text { (inches) }\end{array}$ & $\begin{array}{c}\text { Distal OD } \\
(\mathrm{Fr})\end{array}$ & $\begin{array}{c}\text { Usable length } \\
(\mathrm{cm})\end{array}$ \\
\hline Marathon & 0.0130 & 1.5 & 165 \\
\hline Headway Duo & 0.0165 & 1.6 & $156 / 167$ \\
\hline Prowler 10 & 0.0150 & 1.7 & 150 \\
Excelsior SL-10 & 0.0165 & 1.7 & 150 \\
\hline Echelon 10 & 0.0170 & 1.7 & 150 \\
\hline Headway 17 & 0.0170 & 1.7 & 150 \\
\hline
\end{tabular}

Abbreviations: ID, inner diameter; OD, outer diameter. may limit the use of certain detachment systems but fortunately with the use of the cable system from the Barricade coil system detachment was possible.

Lastly, the relatively decreased braiding of the Marathon increases the susceptibility to kinking or buckling [5]. This can have effects on microcatheter stability in the aneurysm dome during removal of the micro guidewire, advancing it across the interstices of a stent, or event during deployment of coils. Care must be taken to minimize the microcatheter bends as well as maintaining near continuous anterograde saline flush.

Table 1 demonstrates a comparison of the distal ID, OD and length of various microcatheters and shows how the Marathon compares to the smallest microcatheters currently available for coil embolization. The distal ID is at least 0.0020 " smaller, distal OD is smaller, and the length $15 \mathrm{~cm}$ longer. Fortunately, when evaluating the wire diameter, the majority of coils are not larger than 0.0120 ", which should accommodate the distal ID of the Marathon of 0.0130".

The lengths of the pusher wires for each of the coils are shown in Table 2, and only the Target coil appears to be $10 \mathrm{~cm}$ longer. In our third case though, the RHV was still removed to safely use the detachment device. During cessation of heparinized flush during detachment, there is a risk for thrombus formation within the microcatheter. In our second case, thrombosis of the microcatheter lumen interfered with additional coil deployment. Meticulous attention to maintaining continuous flush as much as possible may minimize this risk.

The obvious implication of this report is the possibility of using coils in addition to a liquid embolic agent in the embolization of brain or spinal arteriovenous malformations. Potentially, a small coil mass could be deployed, the microcatheter then advanced past this, and the coil mass could serve as a catalyst for a plug to be formed behind the microcatheter. This could significantly shorten the time needed to create a sufficient plug and perhaps reduce the risks associated with

Table 2. Comparison of Relevant Dimensions Among 10-size Coils Used for Aneurysm Embolization

\begin{tabular}{lcc}
\hline Coil & Wire diameter (inches) & Length $(\mathrm{cm})$ \\
\hline Barricade & $0.0100-0.0120$ & 175 \\
Target & $0.0095-0.0120$ & 185 \\
Axium & $0.0115-0.0125$ & 175 \\
Orbit & $0.0120-0.0140$ & 175 \\
Cosmos & $0.0100-0.0120$ & 175 \\
\hline
\end{tabular}


David A. Stidd, et al.

excessive reflux.

Another implication would be for embolization of very small presumably ruptured aneurysms. Doerfler et al [6]. reported the procedural rupture rate to be five times higher with aneurysms $<3 \mathrm{~mm}$. Oftentimes in these cases, the tip of a 1.7-Fr microcatheter occupies a significant portion of the dome of the small aneurysm, with little room for the coil to be inserted and assume its preformed shape. The smaller distal OD and the overall softer distal segment of the Marathon microcatheter may allow for more room in the small aneurysm dome for the coil to form its shape instead of pushing against the aneurysm wall.

The use of the Marathon microcatheter for coil embolization is feasible, and introduces the possible role and advantage of having a smaller and longer microcatheter for unique challenges during aneurysm embolization. Although not designed or approved to accommodate coils, it is possible, and may be of benefit in technically challenging situations.

\section{References}

1. Mascitelli JR, Polykarpou MF, Patel AA, Kamath AA, Moyle H, Patel AB. Initial experience with Penumbra Coil 400 versus standard coils in embolization of cerebral aneurysms: a retrospective review. J Neurointerv Surg 2013;5:573-576

2. Zoarski GH, Mathis JM, Hebel JR. Performance characteristics of microcatheter systems in a standardized tortuous pathway. AJNR Am J Neuroradiol 1998;19:1571-1576

3. Blanc R, Piotin M, Mounayer C, Spelle L, Moret J. Direct cervical arterial access for intracranial endovascular treatment. Neuroradiology 2006;48:925-929

4. Horie N, Hayashi K, Morikawa M, Nagata I. Selective coil embolization through flow-directed microcatheter for intracranial arteriovenous malformations. Acta Neurochir (Wien) 2012;154: 989-991

5. Kiyosue H, Hori Y, Matsumoto S, Okahara M, Tanoue S, Sagara $\mathrm{Y}$, et al. Shapability, memory, and luminal changes in microcatheters after steam shaping: a comparison of 11 different microcatheters. AJNR Am J Neuroradiol 2005;26:2610-2616

6. Doerfler A, Wanke I, Egelhof T, Dietrich U, Asgari S, Stolke D, et al. Aneurysmal rupture during embolization with Guglielmi detachable coils: causes, management, and outcome. AJNR Am J Neuroradiol 2001;22:1825-1832 